\title{
Drogodependientes vs. usuarios de salud mental con trastornos de personalidad: su relación con la calidad de vida, la psicopatología en Eje I, el ajuste psicológico y dinámica familiar
}

\author{
José Miguel Martínez-González ${ }^{1 *}$, Pilar Munera-Ramos² y Elisardo Becoña-Iglesias ${ }^{3}$ \\ ${ }^{1}$ Centro Provincial de Drogodependencias de Granada, España \\ 2 Hospital Clínico de Granada, España \\ ${ }^{3}$ Universidad de Santiago de Compostela, España
}

\begin{abstract}
Resumen: El objetivo de este estudio ha sido analizar algunas variables de gran relevancia en el tratamiento de los trastornos de la personalidad como son la calidad de vida, el nivel de gravedad de ajuste psicológico, la concomitancia con psicopatología del Eje I y la percepción de dinámica familiar de estos pacientes.

La muestra la componen 68 pacientes que presentan un trastorno de la personalidad de los que el $57.4 \%$ son drogodependientes en tratamiento en un centro ambulatorio de atención a las drogodependencias y el $42.6 \%$ son usuarios de un centro ambulatorio de salud mental que no presenta trastornos adictivos.

Los resultados muestran que no existen diferencias significativas en calidad de vida ni gravedad de ajuste psicológico entre los pacientes en función de la drogadicción. Las diferencias parecen estar vinculadas a la concomitancia de psicopatología en el Eje I, porque los que no la presentan cuentan con un nivel de calidad de vida más elevado y mejor ajuste psicológico. Esto indica que la presencia de psicopatología en el Eje I, en pacientes con trastornos de la personalidad, incrementa la dificultad asociada al tratamiento de estos pacientes. En relación a la dinámica familiar se ha podido constatar que mientras el apego influye negativamente en la calidad de vida de estos pacientes, los recursos familiares la mejoran.

Todo parece indicar que la calidad de vida autopercibida en pacientes con trastornos de la personalidad, drogodependientes o usuarios de salud mental, está determinada por la presencia concomitante de otra psicopatología en el Eje I. Esta concomitancia debe convertirse en objetivo de intervención terapéutica.

Palabras clave: Trastornos de la personalidad; drogodependientes; salud mental; calidad de vida; dinámica familiar.
\end{abstract}

\section{Introducción}

El concepto de calidad de vida (en adelante CV) en el ámbito de la salud mental está asociado a modelos de intervención que buscan mejorar las condiciones de vida de las personas con trastornos psicológicos, teniendo en cuenta sus necesidades individuales (Verdugo, 2002). Particularmente en drogodependientes se viene utilizando desde hace años como indicador de los resultados de la intervención terapéutica (Iraurgi, 2002,). La Organización Mundial de la Salud (THE WHOQOL Group, 1995) define la CV como: "la percepción del individuo de su situación en la vida, dentro del contexto cultural y de los valores en que vive, y en relación con sus objetivos, expectativas, valores e intereses" (p. 1405). Aunque puede medirse de diversas maneras, mayoritariamente se piensa que al menos se deben tener en cuenta en su evaluación los dominios físico, psicológico y social (Katschning, Freeman, y Sartorius, 2000; Wiessma, 2000;).

* Dirección para correspondencia [Correspondence address]: José Miguel Martínez González. Centro Provincial de Drogodependencias de Granada. Hospital de San Juan de Dios. C / San Juan de Dios, 11. 18001, Granada (España). E-mail: jimmgonz@dipgra.es
Title: Addicts vs mental health users with personality disorders: its relation to quality of life in Axis I psychopathology, psychological adjustment and family dynamics.

Abstract: The aim of this study was to analyze some variables of great relevance in the treatment of personality disorders such as quality of life, the severity level of psychological adjustment, the concomitant Axis I psychopathology and perception of family dynamics of these patients.

The sample is composed of 68 patients with a personality disorder of which $57.4 \%$ are drug addicts undergoing treatment in an outpatient drug addiction center and $42.6 \%$ are users of a mental health clinic has not addictive disorders.

The results showed no significant differences in life quality or severity of psychological adjustment among patients on the basis of drug addiction. The differences appear to be linked to the concomitance of psychopathology on Axis I, because for those who do not have a quality of life higher and better psychological adjustment. This indicates that the presence of Axis I psychopathology, in patients with personality disorders, increases the difficulty associated with treating these patients. In terms of family dynamics has been shown that while the attachment has a negative impact on quality of life of these patients, family resources the better.

It seems that the perceived quality of life in patients with personality disorders, drug or mental health consumers, is determined by the concomitant presence of other psychopathology on Axis I. This concomitant should be targeted for therapeutic intervention.

Key words: Personality disorders; drug addicts; mental health; quality of life and family dynamics.

Los estudios que han analizado la $\mathrm{CV}$ en pacientes con TP concluyen que la psicopatología en el Eje II influye negativamente sobre dicha $\mathrm{CV}$, porque se sabe que el carácter de las personas juega un papel importante en la manera en que se percibe la CV (Fassino, Abbate, Delsedime, Rogna, y Boggio, 2004; Martínez-González, Graña y Trujillo, 2011), por lo que los TP pueden ser un destacado condicionante del nivel de CV autopercibido de nuestros pacientes. La influencia de la personalidad en la CV viene dada fundamentalmente por el número de criterios confirmados de TP, por lo que existe una relación lineal entre el número de estos criterios y el nivel de CV percibida (Torgersen, 2007).

Los pacientes con TP, a diferencia de los que no presentan esta psicopatología, perciben menor bienestar subjetivo y de autorrealización, tienen más problemas relacionales, mayor frecuencia de acontecimientos negativos (Torgensen, 2007), peores trabajos y deficientes relaciones sociales (Andreoli, Gressot, Aapro, Tricot y Gognalsons, 1989) y, sobre todo, muestran peores niveles de ajuste social en comparación con los que presentan psicopatología sólo en el Eje I (Narud, Mykletun y Dahl, 2005).

Por otro lado, el nivel de CV percibida puede incluso diferir dependiendo del tipo de TP (Cramer, Torgersen y 
Kringlen (2006). En el estudio de Cramer, et al. (2006) se encontraron peores niveles de CV en los TP, y por este orden, de evitación, límite, esquizotípico, dependiente, paranoide, esquizoide y antisocial. Por el contrario, se observó mejor CV en pacientes diagnosticados de TP histriónico y narcisista. Además, los pacientes con TP obsesivocompulsivo sólo muestran una discreta reducción de la $\mathrm{CV}$, cosa que no sucede con el resto de TP (Pedrero, Olivar y Chicharro, 2008).

Además, la evaluación de la $\mathrm{CV}$ en pacientes con psicopatología debe hacerse teniendo en cuenta también otras variables, porque el bienestar subjetivo refleja los estados psicopatológicos alterados (Atkinson, Zibin, y Chuang, 1997). Esto significa que las personas utilizan su estado afectivo momentáneo como referencia para establecer juicios sobre su felicidad y satisfacción en la vida, de modo que las medidas de CV correlacionan considerablemente con el Eje I (Lozano, Rojas, Pérez, González-Saiz, Ballesta e Izaskum, 2008). Se constata que la psicopatología en el Eje I influye en la percepción de la calidad de vida. Así, puede decirse que la gravedad del ajuste psicológico (en adelante GAP) es un buen predictor de la calidad de vida autopercibida en drogodependientes (Martínez-González, Graña y Trujillo, 2010).

Los objetivos de este trabajo son, en personas que tienen TP, dos: 1. Conocer las diferencias en CV, GAP y percepción de la dinámica familiar entre dos grupos de pacientes que siguen tratamiento a nivel ambulatorio, por un lado, usuarios de una Unidad de Salud Mental Comunitaria y por otro, usuarios de un Centro de Tratamiento de Drogode- pendencias; y 2. Indagar en la importancia de la coexistencia del Eje II en estas poblaciones.

\section{Método}

\section{Participantes}

La muestra la componen 68 personas que presentan un TP, de los que el $57.4 \%(n=39)$ son drogodependientes en tratamiento en el Centro Provincial de Drogodependencias de Granada y el $42.6 \%(n=29)$ pacientes en tratamiento en el Centro Ambulatorio de Salud Mental de Baza (Granada). En el grupo de participantes usuarios de Salud Mental se descartó adicción a sustancias y adicción sin sustancias. Los pacientes drogodependientes no presentaron en ningún caso diagnóstico de adicción sin sustancias.

El $54.4 \%(n=37)$ son hombres y el $45.6 \%(n=31)$ mujeres. En el grupo de drogodependientes el $71.79 \%$ ( $\mathrm{n}=$ 28) eran hombres, mientras que el $28.20 \%(n=11)$ eran mujeres. En el grupo de usuarios de salud mental el $31.03 \%$ (n $=9)$ eran hombres, mientras que el $68.96 \%(n=20)$ mujeres. La edad media de los participantes es de 42.60 años, con un rango que va de los 18 a los 87 años. El $68.2 \%(\mathrm{n}=45)$ vive con su familia propia o pareja mientras que el 28.8. \% $(n=19)$ vive con su familia de origen. Solo hay dos personas que viven solas.

La distribución de la psicopatología tanto en el Eje I como en el Eje II puede verse en la Tabla 1 y Figura 1.

Tabla 1. Psicopatología en el eje I y eje II de todos los sujetos (DSM-IV-TR).

\begin{tabular}{|c|c|c|c|c|c|c|}
\hline \multirow{2}{*}{$\frac{\text { Eje II }}{\text { Paranoide }}$} & \multicolumn{2}{|c|}{ Frecuencia \% } & $\mathrm{N}$ drogodependientes & \multirow[t]{2}{*}{$\%$} & \multirow{2}{*}{$\frac{\mathrm{N} \text { usuarios de salud mental }}{2}$} & \multirow{2}{*}{$\begin{array}{l}\% \\
6.9\end{array}$} \\
\hline & 2 & 2.9 & & & & \\
\hline Esquizoide & 1 & 1.5 & & & 1 & 3.4 \\
\hline Histriónico & 4 & 5.9 & 2 & 5.1 & 2 & 6.9 \\
\hline Límite & 6 & 8.8 & 3 & 7.7 & 3 & 10.3 \\
\hline Obsesivo-compulsivo. & 27 & 39.7 & 21 & 53.8 & 6 & 20.7 \\
\hline Dependencia & 15 & 22.1 & 9 & 23.1 & 6 & 20.7 \\
\hline Evitación & 5 & 7.4 & 1 & 2.6 & 4 & 13.8 \\
\hline No especificado & 8 & 11.8 & 3 & 7.7 & 5 & 17.1 \\
\hline Total & 68 & 100.0 & 39 & 100 & 29 & 100 \\
\hline \multicolumn{7}{|l|}{ Eje I } \\
\hline No presenta Eje I & 34 & 47.6 & 30 & 74.3 & 5 & 17.5 \\
\hline $\mathrm{T}$ ansioso - depresivo & 13 & 20.6 & 2 & 7.6 & 12 & 41.4 \\
\hline Depresión & 7 & 11.1 & 4 & 10.3 & 2 & 6.9 \\
\hline T de estado de ánimo no especificado & 1 & 1.6 & 1 & 2.6 & & \\
\hline Fobia social & 2 & 3.2 & 1 & 2.6 & 4 & 13.8 \\
\hline T distímico & 5 & 7.9 & 1 & 2.6 & 1 & 3.4 \\
\hline T somatomorfo & 1 & 1.6 & & & 1 & 3.4 \\
\hline T alimentación (Bulimia) & 1 & 1.6 & & & 1 & 3.4 \\
\hline $\mathrm{T}$ ciclotímico. & 1 & 1.6 & & & 1 & 3.4 \\
\hline Síndrome ansioso & 1 & 1.6 & & & 1 & 3.4 \\
\hline TOC & 1 & 1.6 & & & 1 & 3.4 \\
\hline Total & 63 & 100.0 & 39 & 100 & 29 & 100 \\
\hline Drogodependientes & \multicolumn{6}{|c|}{ Usuarios de Salud Mental } \\
\hline$\overline{\mathrm{NO} \text { presenta Eje I }}$ & \multicolumn{4}{|c|}{ NO presenta Eje I } & SI presenta Eje I & \\
\hline $\mathrm{n}$ & $\%$ & $\mathrm{n}^{1}$ & $\% \quad \mathrm{n}$ & $\%$ & $\mathrm{n}$ & $\%$ \\
\hline 30 & $76.9 \%$ & 9 & $23.1 \% 5$ & $17.2^{\circ}$ & 24 & $82.8 \%$ \\
\hline
\end{tabular}




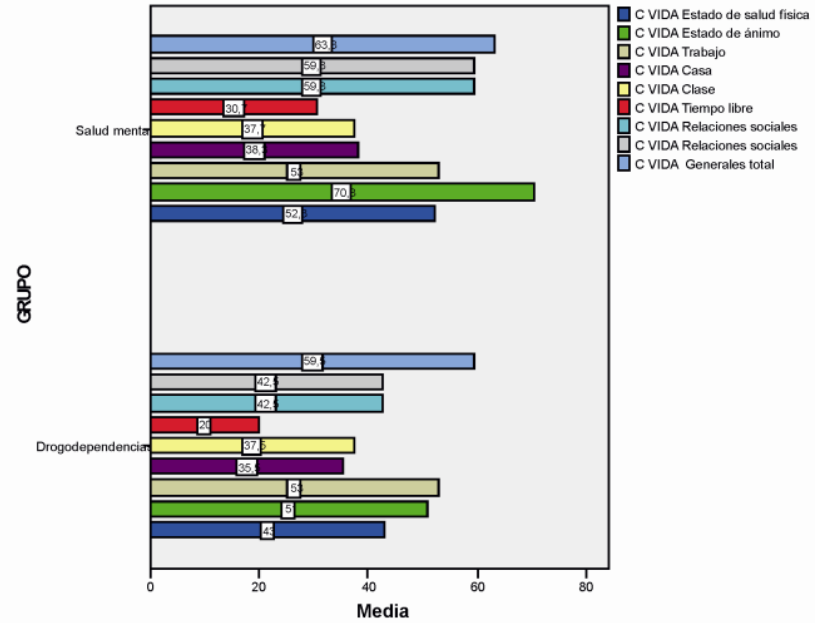

Figura 1. Áreas del cuestionario de calidad de vida en drogodependientes y usuarios de salud mental.

En cuanto al tiempo que lleva cada paciente en tratamiento, la muestra la componen desde pacientes que aún no llevaban un mes hasta los que llevaban 36 meses. El tiempo medio de tratamiento en el momento de la evaluación fue de 5.65 meses $(D . T .=6.81)$.

\section{Instrumentos}

Para la medición de la CV se utilizó el Cuestionario de Calidad de Vida: Sastisfacción y Placer (Quality of Life Enjoyment and Satisfaction Questionnaire, Q-LES-Q) (Endicott, Harrison y Blumenthal, 1993). Este cuestionario mide de forma sensible la satisfacción y el placer del paciente en su vida cotidiana. Es un instrumento genérico de $\mathrm{CV}$ aplicable a todo tipo de pacientes, con independencia de su diagnóstico y tratamiento. Consta de 93 ítems que exploran las siguientes ocho áreas: estado de salud física/actividades, estado de ánimo, trabajo, actividades del hogar, tareas de clase, actividades de tiempo libre, relaciones sociales y actividades generales. La consistencia interna de todas las áreas es aceptable (Martínez-González, Graña y Trujillo, adicciones). El cuestionario incorpora al final un apartado resumen para que la persona haga una valoración general de cada área. Las respuestas son de tipo Likert de cinco alternativas de respuesta. Las puntuaciones altas indican buena $\mathrm{CV}$, mientras que las bajas representan una mala $\mathrm{CV}$. El marco de referencia temporal para contestar al cuestionario es la semana pasada.

Para la medición de presencia de psicopatología no psicótica se utilizó el General Health Questionnarie - $G H Q$ 28 (Goldberg y Hiller, 1979), que establece un índice de gravedad de ajuste psicológico (GAP) construido a partir de la suma de cuatro subescalas: síntomas somáticos, ansiedad, disfunción social y depresión. (González-Saiz, Carulla, Martínez, López, Ruiz y Guerra, 1997).

Para la evaluación y diagnóstico de los TP se utilizó la entrevista semiestructurada Examen Internacional de los Trastornos de la Personalidad (IPDE) (Loranger, 1995; versión españo- la de López-Ibor, Pérez Urdániz y Rubio, 1996) Dispone de un screening con respuestas de verdadero-falso, y una entrevista clínica que matiza la presencia o no de TP según criterios DSM.

La evaluación del Eje I se llevó a cabo a través de entrevista clínica, atendiendo a criterios DSM-IV (APA, 2002). Para confirmar la presencia de psicopatología en el Eje, I se utilizó la SCID-I (First, Spitzer, Gibbon y Williams, 1999).

Para completar la evaluación de la CV se quiso profundizar en la dinámica familiar percibida por el paciente, que se evaluó a través del FAP/FACES-IV (Family Assessment Package / Family Adaptability and Cohesion Evaluation Scale) (Sanz, Iraurgi y Martínez, 2002). El instrumento evalúa el funcionamiento familiar con las subescalas de: estrés familiar, comunicación familiar, recursos familiares, satisfacción familiar, desapego, apego, rigidez y caos. La respuesta es de tipo Likert de cinco alternativas.

Para descartar la presencia de trastorno por consumo de sustancias en usuarios de salud mental se utilizó la versión española de la escala Severity of Dependence Scale (GonzálezSaiz, de las Cuevas, Barrio y Domingo-Salvary, 2008).

Con la finalidad de descartar trastornos del control de impulsos y adicciones sin sustancias en el grupo de pacientes de la Unidad de Salud Mental se utilizó el MULTICAGE CAD-4 (Pedrero, Rodríguez, Gallardo, Fernández, Pérez y Chicharro, 2007). Las tareas de diagnóstico psicopatológico y pasación de las pruebas descritas las llevaron a cabo un psicólogo clínico en cada una de los servicios, responsables del tratamiento de las personas que han participado en el estudio. En el grupo de drogodependientes se realizó cuando los pacientes contaban con al menos quince días de abstinencia.

\section{Diseño y análisis estadístico}

Se empleó una metodología cuasi-experimental, concretada ésta en distintos diseños unifactoriales entre grupos. Junto a un análisis descriptivo de las variables clínicas de interés en el estudio, se realizaron análisis de contrastes sobre diferencias de medias mediante el estadístico t de Student, habiéndose establecido un nivel de significación para tomar decisión estadística de 0.05 . El análisis se realizó con el programa SPSS 15.0. Además se realizaron diferentes ANOVAS para indagar en los efectos principales y de interacción de las diferentes variables independientes sobre la variable dependiente, así como análisis de regresión para conocer la capacidad predictiva de algunas variables.

\section{Procedimiento}

En la Unidad de Salud Mental se realizó una selección de los pacientes con TP que estaban en tratamiento durante un periodo de tiempo, mientras el grupo de pacientes drogodependientes se configuró escogiendo los pacientes de una muestra más amplia en función del tiempo que llevaban en tratamiento con la intención de homogeneizar los dos grupos. 


\section{Resultados}

\section{Presencia concomitante de Eje I}

Al comparar la presencia de psicopatología en el Eje I concomitante con el TP, se observa que existen diferencias estadísticamente significativas en función de si el paciente es drogodependiente o es usuario de salud mental, siendo mayor el número de personas que presentan esta concomitancia en el segundo grupo $(t=-5.946 ; p<.001 ; d=-2.3989)$, es decir, son más los usuarios de salud mental que presentan de forma concomitante al TP psicopatología en el Eje I no relacionada con el consumo de sustancias. En el grupo de usuarios de salud mental el $82.8 \%$ presentan psicopatología en el Eje I, mientras que en el grupo de drogodependientes solo un $23.1 \%$. En la Tabla 1 puede verse la distribución de psicopatología en la muestra.

\section{Calidad de vida}

En todas las áreas evaluadas del cuestionario de calidad de vida, los usuarios de salud mental presentan puntuaciones mayores, aunque las diferencias no son estadísticamente significativas (Figura 1). Según esto, los pacientes con TP que no presentan a su vez una drogadicción refieren niveles de calidad de vida mayores a los que describen los pacientes que presentan una drogodependencia. También se compararon los apartados generales del cuestionario de calidad de vida entre los dos grupos no observándose diferencias estadísticamente significativas, lo que muestra que los niveles de calidad de vida no dependen tanto de la presencia de la drogadicción como de los TP en sí mismos.

Se realizaron diferentes ANOVAS para conocer los efectos principales y de interacción de algunas variables independientes (VI) de interés clínico sobre la variable dependiente (VD) nivel de CV autopercibido. Por un lado se tuvieron en cuenta las VI: género y presencia de psicopatología en el Eje I de forma concomitante al TP, con dos niveles: presencia y no presencia de Eje I. El análisis muestra significatividad del efecto de la interacción de las dos VI sobre la $\operatorname{VD}\left(F_{1-68}=4.741 ; p=.033 ;\right.$ Eta parcial al cuadrado $\left.=.070\right)$. También se obtiene efecto significativo de la variable presencia de Eje I $\left(F_{1-68}=10.395 ; p=.002\right.$; Eta parcial al cua$\mathrm{drado}=.142)$, lo que indica que el nivel de CV percibido viene determinado por la presencia de psicopatología concomintante

En un segundo análisis se tuvieron en cuenta las VI: grupo, con dos niveles - grogodependientes y pacientes de la unidad de salud mental-; y la variable presencia de Eje I, con dos niveles. Los resultados muestran también significatividad de la VI presencia de Eje I $\left(F_{1-68}=7.618 ; p=.008\right.$; Eta parcial al cuadrado $=.108)$, no observándose en la variable grupo ni en la interacción de ambas variables.

En el tercer análisis se incluyeron dos VI: edad, con dos niveles - menores de 40 años y mayores de 40 años-; y presencia de Eje I, con dos niveles. En este caso se observa sig- nificación tanto en efecto principal de la presencia de Eje I $\left(F_{1-68}=7.407 ; p=.008\right.$; Eta parcial al cuadrado $\left.=.008\right)$ como de la interacción de ésta variable y la edad $\left(F_{1-68}=7.121 ; p=\right.$ .010 ; Eta parcial al cuadrado $=1.02$ ).

\section{Ajuste psicológico}

En relación a los niveles de ajuste psicológico, medidos a través del cuestionario GHQ-28, no se obtienen diferencias estadísticamente significativas entre los dos grupos de pacientes.

El estudio de la relación entre el nivel de ajuste psicológico y la calidad de vida autopercibida muestra que el ajuste psicológico predice el nivel de calidad de vida (en su escala general) en sentido inverso, de modo que a mayor GAP peor calidad de vida percibe el paciente con TP $\left(\mathrm{R}^{2}=.344 ; B e-\right.$ $t a=-.856 ; p<.001)$. Por el contrario, el tiempo que llevan los pacientes en tratamiento por el contrario no tiene capacidad para predecir el nivel de CV.

En relación a la dinámica familiar se observa que el nivel de GAP predice el nivel de estrés familiar en sentido positivo $\left(R^{2}=124 ;\right.$ Beta $\left.=.352 ; p<.01\right)$ y el nivel de satisfacción familiar en sentido negativo $\left(R^{2}=.079 ;\right.$ Beta $=-.281 ; p<$ .05), de modo que a medida que aumenta la GAP empeora la satisfacción percibida en la dinámica familiar y aumenta el estrés familiar de los pacientes con TP. Por otra parte, el nivel de GAP no tiene capacidad de predecir el resto de áreas del cuestionario de dinámica familiar.

\section{Dinámica familiar}

En la tabla 2 puede observarse el perfil de esta muestra en relación a escalas y dimensiones del cuestionario de dinámica familiar. La duración del tratamiento no correlaciona con ninguna de las escalas del cuestionario FAPFACES-IV. Tampoco se obtienen diferencias estadísticamente significativas en las dimensiones del cuestionario, en función del grupo, esto es, drogodependientes o usuarios de salud mental. Además no se encuentran diferencias estadísticamente significativas en función de la presencia de psicopatología concomitante en el Eje I.

Tabla 2. Puntuaciones de las escalas y dimensiones del FAP-FACES de la totalidad de la muestra.

\begin{tabular}{lllll}
\hline & Mínimo & Máximo & Media & DT \\
\hline Estrés Familiar. & 1 & 4 & 2.19 & .736 \\
Comunicación. & 1 & 5 & 3.26 & .843 \\
Recursos familiares. & 1 & 5 & 3.35 & .744 \\
Satisfacción familiar. & 1 & 5 & 3.36 & .954 \\
Desapego. & 1 & 5 & 2.31 & .802 \\
Apego. & 1 & 4 & 2.55 & .626 \\
Rigidez. & 1 & 4 & 2.50 & .687 \\
Caos. & 1 & 5 & 2.33 & .891 \\
Dimensión: cohesión. & 1 & 4 & 2.43 & .515 \\
Dimensión: adaptabilidad.1 & 5 & 2.43 & .634
\end{tabular}




\section{Dinámica familiar y calidad de vida}

Se han realizado diferentes regresiones lineales para conocer la capacidad predictiva de la dinámica familiar sobre la percepción de calidad de vida analizada a través del área de valoración general. Ni la comunicación ni el estrés familiar tienen capacidad predictiva sobre la $\mathrm{CV}$, pero sin embargo sí tiene capacidad predictiva los recursos familiares $\left(R^{2}=.170\right.$; $B$ eta $=.412 ; p<.001)$. Por tanto, los recursos familiares influyen en el nivel de CV autopercibida de los pacientes con TP, de modo que a mayor disponibilidad de recursos más elevado es el nivel de CV autopercibido.

$\mathrm{El}$ análisis de correlaciones bivariadas de la escala de valoración general de CV y las diferentes escalas del cuestionario FAP-FACES-IV, muestra que los recursos familiares correlacionan de forma positiva y estadísticamente significativa con la CV $(r=.412 ; p<.001)$ mientras que el apego correlaciona de forma negativa con la escala de valoración general de CV $(r=-.253 ; p<.05)$.

La dimensión cohesión predice en sentido negativo la CV general $\left(R^{2}=.085 ;\right.$ Beta $\left.=-.291 ; p<.05\right)$. Por otro lado, el análisis de regresión muestra que las escalas de recursos familiares y apego predicen el nivel de CV general aunque no en el mismo sentido. Los recursos familiares predicen en sentido positivo $\left(R^{2}=.170 ;\right.$ Beta $\left.=.412 ; p<.001\right)$ y el apego en sentido negativo $\left(R^{2}=.064\right.$; Beta $\left.=-.253 ; p<.05\right)$. En análisis de regresión múltiple muestra que los recursos familiares tienen más peso en la predicción, lo que supondría que los recursos podrían contrarrestar el impacto del apego sobre la percepción de la CV de los pacientes con TP.

\section{Discusión}

Esta investigación nos ha permitido profundizar en los objetivos propuestos. La incidencia de psicopatología en el Eje I que se obtiene es coincidente con otros estudios realizados con muestras de drogodependientes con TP (Skodol, 2007). Esto muestra el hecho de que las personas con TP no acuden a los recursos asistenciales, por regla general, demandando ayuda para superar el TP, sino que lo hacen como consecuencia del malestar que genera la presencia de psicopatología en el Eje I concomitante. En drogodependientes es la adicción específicamente la que causa egodistonía, mientras que en usuarios de salud mental esta función la ocupa la psicopatología en el Eje I.

Por otro lado el alto porcentaje de psicopatología concomitante en TP debe alertarnos sobre el posible solapamiento de sintomatología del Eje I con los TP. La IPDE es una entrevista estructurada que afronta satisfactoriamente la contaminación entre Ejes (Loranger, Lenzenweger, Gartner, Lehmann, Herzig, Zammit, Gartner, Abrams, y Young, 1991). Además, la convierte, a nuestro juicio, en el instrumento idóneo para la evaluación y diagnóstico de los TP cuando coexisten con psicopatología en el Eje I. Por otro lado, esta concomitancia podría explicar, al menos en parte, las dificultades asociadas al tratamiento dispensado en aten- ción primaria a algunas personas, que como se sabe es la puerta de entrada de muchas demandas. En atención primaria se reiteran frecuentemente demandas asistenciales que no remiten fácilmente y que podrían estar vinculadas con la psicopatología del Eje II.

Se sabe que los pacientes con TP presentan niveles de CV más bajos en comparación a aquellos que no presentan esta psicopatología. No se han encontrado diferencias entre los pacientes con TP dependiendo de si pertenecen al grupo de drogodependientes o usuarios del dispositivo de salud mental, lo que hace pensar que es el carácter la variable de mayor influencia en la percepción de la CV y no tanto la drogadicción, relación que ha sido demostrada en otros estudios (Torgersen, 2007).

En relación al impacto que puede tener la presencia de Eje I en pacientes con TP en la CV autopercibida, los resultados de este trabajo coinciden con los hallados en la investigación de Narud, et al., (2005). Todo parece indicar que la calidad de vida autopercibida en pacientes con TP es menor cuando el paciente presenta psicopatología en el Eje I. Esta relación se evidencia también en otros trabajos que han vinculado el nivel de GAP con el nivel de CV (MartínezGonzález, et al., 2010). Por todo esto, puede decirse que la presencia de esta psicopatología influye negativamente en la CV autopercibida de los pacientes con TP. De los resultados del estudio puede concluirse que el tratamiento de pacientes con TP es más laborioso cuando coexisten con psicopatología en el Eje I. Las intervenciones deben cuidar particularmente el curso de la psicopatología del Eje I (MartínezGonzález. 2011).

Los resultados muestran que el nivel de CV percibido no depende tanto de si drogodependiente o no, sino de la presencia de psicopatología comórbida del Eje I en ambos grupos de pacientes. Esta variable presenta significación en cada una de las ANOVAS realizadas, lo que la convierte en un objetivo de intervención terapéutica de primera magnitud en pacientes que presentan un TP.

En relación a la dinámica familiar, se ha constatado que el apego y la dimensión cohesión tienen capacidad de predecir en sentido negativo el nivel de CV autopercibido, lo que denota la importancia que tiene la percepción de la dinámica familiar de estos pacientes. El hecho de que un mayor apego correlacione de forma negativa con la $\mathrm{CV}$ demuestra que una interacción más estrecha entre los miembros de la familia somete probablemente al paciente a una situación de constante exposición de sus deficientes habilidades relacionales, toda vez que se sabe que las personas con TP presentan diferencialmente menos capacidad empática y más dificultad para relacionarse con otras personas. Estos resultados nos indican la necesidad de cuidar particularmente el tipo de relación familiar que queremos para cada paciente, porque según esto, en algunos casos el distanciamiento del paciente con sus progenitores podría favorecer la evolución del paciente. La experiencia muestra que esto sucede con algunos pacientes con TP y particularmente en pacientes con trastorno límite de la personalidad (Gunderson, 2002). También, 
debe contemplarse en las intervenciones la potenciación de los recursos familiares y la satisfacción general, teniendo en cuenta que son escalas que predicen un mayor nivel de CV. Probablemente los pacientes que perciben a sus familias con mayor nivel de recursos las perciben con capacidad de prever los conflictos entre los miembros de la familia y en general con más capacidad para gestionar las situaciones de conflicto (Iraurgi, Sanz y Martínez-Pampliega, 2004). Los recursos amortiguan los problemas derivados de las interacciones entre los miembros de la familia (Martínez- González, 2010).

Las intervenciones familiares deberían cuidar particularmente las estrategias que persiguen mejorar el apego de estos pacientes con sus familiares, dado que como consecuencia de la dificultad del paciente para las interacciones con otros, se le podría exponer a interacciones para las que no cuentan con las habilidades necesarias. Parece claro que los familiares

\section{Referencias}

APA, (2002). DSM-IV-TR Manual diagnóstico y estadístico de los trastornos mentales, IV Texto Revisado. Barcelona: Masson.

Andreoli, A., Gressot, G., Aapro, N., Tricot, L. y Gognalsons, M. (1989). Personality disorders as a predictor of outcome. Journal of Personality Disorder, 3, 307-321.

Atkinson, M., Zibin, S. y Chuang, H. (1997). Characterizing Quality of life among patients with chronic mental illness: A critical examination of the self-reort methodology. American Journal of Psychiatry, 105, 99-105.

Cramer, V., Torgersen, S., y Kringlen, E. (2006). Personality disorders and quality of life. A population study. Comprehensive Psychiatry, 47, 178-184.

Endicott, J., Harrison, N.W. y Blumenthal, W. 1993. Quality of Life Enjoyment and Satisfaction Questionnaire: A new Measure. Psychopharmacoogical Bulletin, 29, 321-326.

Fassino, S., Abbate, G., Delsedime, N., Rogna, L., y Boggio, S. (2004). Quality of life and personality disorders in heroin abusers. Drug and Alcohol Dependence, 76, 73-80.

First, M., Spitzer, R., Gibbon, M. y Williams, J. (1999). Guia del usuario para la entrevista clinica estructurada para los trastornos de la personalidad del Eje I del DSM-IV (SCID-I). Madrid: Masson.

González-Saiz, F.M., Carulla, S., Martínez J.M., López, A., Ruiz, J. y Guerra, D. (1997). Indicador del tratamiento de la adicción a opiáceos. Cádiz: Servicio de Publicaciones de la Universidad de Cádiz.

González-Saiz, F., de las Cuevas, C., Barrio, G. y Domingo-Salvary, A. (2008). Versión española consensuada de la Severity of Dependence Scale (SDS). Medicina Clinica, 131, 796-799.

Golberg, D.P. y Hiller, V.F. (1979). A scaled version of the General Health Questionaire. Windsor: NFER Publishing Company.

Gunderson, J.G. (2002). Trastorno limite de la personalidad. Guía clínica. Barcelona: Ars Médica.

Iraurgi, I., (2002). Instrumentos de evaluación de la calidad de vida relacionada con la salud en toxicomanías. En I. Iraurgi y F. González (Eds.), Instrumentos de evaluación en drogodependencias (pp. 481-511). Barcelona: Aula Médica.

Karow, A., Verthein, V., Krausz, M. y Schäfer, I. (2008). Association of personality disorders family conflicts and treatment with quality of life in opiate addiction. European Addiction Research, 14, 38-46.

Katschning, H., Freeman, H. y Sartorius, N. (2000). Utilidad del concepto de calidad de vida en psiquiatría. En H. Katschning, H. Freeman y N. Sartorius, (Eds.), Calidad de vida en los trastornos mentales (pp. 3-15). Barcelona: Masson.

López-Ibor, J., Pérez, A., y Rubio, V. (1996). Examen Internacional de los Trastornos de la Personalidad; Módulo DSM-IV. Versión Española. Madrid: Organización Mundial de la Salud.

Loranger, A, Lenzenweger, M. Gartner, A., Lehmann, V, Herzig, J., Zammit, G, Gartner, J., Abrams, R. y Young, R. (1991). Trait State asrtifacts and the diagnosis of personality disorders. Archives of General Psychiatry, 48, 720-728.

Lozano, O., Rojas, A., Pérez, C., González-Saiz, F., Ballesta, R., y Izaskum, B. (2008). Evidencias de validez del test para la evaluación de la calidad de vida de los pacientes con TP necesitan de cierto grado de entrenamiento e instrucción para mejorar sus recursos familiares necesarios para afrontar las dificultades que pueden surgir en la convivencia con estos pacientes.

En consonancia con los resultados del trabajo de Karow, et al., (2008) que no hallaron correlación significativa entre el número de conflictos familiares y el nivel de CV, en nuestra investigación hemos podido constatar que la escala de estrés familiar no predice el nivel de CV.

Las limitaciones del presente estudio guardan relación con el tamaño de la muestra, ya que una de mayor tamaño permitiría un mayor número de comparaciones entre las variables utilizadas en el estudio. Aunque el rango de edad de los participantes es muy grande, esta variable se ha introducido como covariable observando que es significativa en su interacción con la presencia de psicopatología en el Eje I.

en adictos a sustancias psicoactivas a partir del modelo biaxial de la adicción. Psicothema, 20, 317-323.

Martínez-González, J.M. (2010). El efecto de relajación en la percepción de la dinámica familiar de pacientes con trastornos de la personalidad. Trastornos Adictivos, 12, 27-32.

Martínez-González, J.M. (2011). Drogodependencias y trastornos de la personalidad: variables relevantes para su tratamiento. Papeles del Psicólogo, 32, 166174.

Martínez-González, Graña y Trujillo, (2010). La calidad de vida en alcohólicos con trastornos de la personalidad: relación con el ajuste psicológico y el $\mathrm{cra}$ ving. Psicothema, 22, 562-567.

Martínez-González, J.M., Graña, J.L. y Trujillo, H. (2011). Estudio longitudinal sobre la calidad de vida, craving y ajuste psicológico en pacientes dependientes del alcohol: variaciones en función de la presencia de trastornos de la personalidad. Adicciones, 23, 227-235

Narud, K., Mykletun, A. y Dahl, A. (2005). Quality of life in patients with personality disorders seen at an ordinary psquiatric outpatient clinic. BMC Psycbiatry, 5, 5. En línea [http://www.biomedcentral.com/1471-244X/5/10].

Pedrero, E., Rodríguez, A., Gallardo, G., Fernández, M., Pérez, M. y Chicharro, J. (2007). Validación de un instrumento para la detección de trastornos de control de impulsos y adicciones: el MULTICAGE CAD-4. Trastornos Adictivos, 9, 269-278.

Pedrero, E., Olivar, A. y Chicharro, J. (2008). Cuestionario CAD-4: una medida biopsicosocial de la calidad de vida autopercibida en pacientes drogodependientes. Trastornos Adictivos, 10, 17-31.

Sanz, M., Iraurgi, I. y Martínez, P. (2002). Evaluación del funcionamiento familiar en toxicomanías: adaptación española y características de adecuación métrica del FAP-FACES-IV. En: I Iraurgi y F González, editores. Instrumentos de evaluación en drogodependencias (p. 403-34). Madrid: Aula Médica.

Skodol, A.E. (2007). Manifestaciones, diagnóstico clínico y comorbilidad. En J.M. Oldham, A. E. Skodol y D. S. Bender (Eds.), Tratado de los trastornos de la personalidad (pp. 59-90). Barcelona: Elsevier Masson.

Torgersen, S. (2007). Epidemiología. En J.M. Oldham, A E Skodol y DS Bender, Tratado de los trastornos de la personalidad (pp. 131-143). Barcelona: Elsevier Masson.

Verdugo, M.A. (2002). Autodeterminación y calidad de vida en salud mental: dos conceptos emergentes. Salud Mental, 25. 68-77.

Wiessma, D. (2000). Desempeño del rol como componente de la calidad de vida de los trastornos mentales. En H. Katschning, H. Freeman N. Sartorius (Eds.), Calidad de vida en los trastornos mentales (pp. 43-52). Barcelona: Masson.

WHOQOL GROUP (1995). The World Health Organization Quality of Life Assessment (WHOQOL): position paper from the World Health Organization. Social Science and Medicine, 41, 1403-1409.

(Articulo recibido: 07-09-2010, revisado: 28-06-2012, aceptado: 15-07-2012) 